\title{
A Two-step Forecasting Solution and Upscaling Technique for Small Size Wind Farms Located in Hilly Areas of Romania
}

\author{
Ion LUNGU' ${ }^{1}$, George CĂRUȚAŞU ${ }^{2}$, Alexandru PÎRJAN², \\ Simona-Vasilica OPREA ${ }^{1}$, Adela BÂRA ${ }^{1}$ \\ ${ }^{1}$ The Bucharest University of Economic Studies, \\ 6 Romană Square, Bucharest, 010374, Romania \\ ion.lungu@ie.ase.ro; simona.oprea@csie.ase.ro; bara.adela@ie.ase.ro \\ ${ }^{2}$ Romanian-American University, \\ 1B Expozitiei Blvd., Bucharest, 012101, Romania \\ carutasu.george@profesor.rau.ro; pirjan.alexandru@profesor.rau.ro
}

\begin{abstract}
Taking into account the well-known benefits of using renewable energy as an important energy source, some practical aspects regarding operation must be underlined. In the specific case of wind power plants (WPP) forecasting the generated wind power is the most important issue for assuring stability of Romania's National Power System (NPS) and economic efficiency of wind power plant operation. The wind power plant operator should report daily to National Dispatching Centre, the hourly energy production for the next day. In case of inaccurate energy production prediction, the National System Operator must take additional measures in order to maintain the stability of NPS. The purpose of our research is to develop a solution, based on Artificial Neural Networks, for forecasting the wind energy production of small output power wind farms located in hilly areas of Romania, thus improving the accuracy of the hourly prediction. The case study is based on two-year historical data for a wind power plant comprising two power production groups situated in Tulcea County, in southeastern Romania. By using this approach, we have also explored the possibility of algorithm generalization, starting from the detailed model of the first production group and generalize it to the second one.
\end{abstract}

Keywords: renewable energy, wind power forecasting, upscaling technique, artificial neural network.

\section{Introduction}

\subsection{Wind energy resources}

In the last years, due to the European legislation facilities for Renewable Energy Source (RES), the wind energy capacity in EU28 has been increasing, up to $128.8 \mathrm{GW}$ [1]. In 2014, 11791 MW were installed, with an estimated value between $€ 13$ bn and $€ 18$ bn. The wind energy share in total energy production is estimated to $285 \mathrm{TWh}$, which represents approximatively $8 \%$ of the total EU energy consumption. Regarding Romania, the same source reveals, at the end of 2014, an installed capacity of $2953.6 \mathrm{MW}$, having risen with 354 MW, when compared to 2013. Concerning the installation rate, the value is nearly half of the previous year, a fact that could be explained by the reduction of energy consumption and green certificates' value. As a whole, the National System Operator (NSO), reported for 1st of July, 2014 an installed capacity of 24582 MW with with the next composition as energy source: Hydro $28.8 \%$, Coal 26.66\%, Hydrocarbon $22.57 \%$, Wind $12.10 \%$, Nuclear $5.74 \%$, Solar 5.15 and Biomass $0.41 \%$. The distribution of energy sources may vary depending upon both on the season and availability. Anyway, the most important annual growth was registered in China [2], 43\%, with a total wind power capacity of $369,597 \mathrm{MW}$.

\subsection{The mechanism of the system}

The National Power System should balance in any moment the energy consumption to production. Because of the hazardous nature of wind, it is difficult to estimate in terms of hours (as a time scale), the wind energy production. The wind power plant operator has to notify the NSO for the next day hourly production. The wind power plant operator receives a subsidy from national government, known as "green certificates".

The value of green certificates and the market mechanism are regulated by EU and national laws $[3,4]$. The actual estimation indicates an accuracy of $70 \%$ for wind power prediction. According to the Commercial Code, if the production is lower than the notification, the wind power plant operator has to pay for the unbalance. The National Dispatching Centre must compensate the unbalance by activating Fast Tertiary Reserves. The higher the prediction error is, the higher the costs 
incurred. The wind power plant operators may use various commercial tools (PredictWind, WPPT, Wind Speed Predictor, WINDcast etc.) developed by independent commercial providers or resulting from international research projects [5]. Our obtained results are part of an integrated system in development [6] as a result of a scientific research project (Intelligent system for predicting, analysing and monitoring performance indicators and business processes in the field of renewable energies - SIPAMER). Due to the field relevance in Romania, the same goal of developing an integrated system for renewable energy sources management is approached by other research projects in progress [7].

The majority of wind farms in Romania have a small output power and are situated on hilly terrain [8]. Therefore, in this paper, we are developing a solution for forecasting the wind energy production and the consumed energy for these types of wind power plants. We aim to improve the forecast accuracy of the existing forecasting software, used by a small wind park located in Baia, in Tulcea County, in South East Romania, by developing a two-step forecasting technique that is best suited for hilly terrain that causes a change of the wind direction from one turbine to another when situated at different altitudes. We also develop an upscaling technique by starting from one turbine and forecasting the energy production for six turbines grouped into two production groups, located on hilly terrain, in close proximity. We focus on obtaining a forecasting solution with an improved accuracy prediction for small wind farms located on hilly terrain and also a solution that can be useful for potential investors that want to build these types of wind farms.

\section{Wind Energy Production Forecast}

\subsection{State of the art}

The actual efforts are focused on improving the accuracy of the wind power prediction. Some research literature is dedicated to wind speed forecasting $[9,10,11]$ and the time scale differs depending on the prediction objective. By analysing these papers, we can distinguish a short-term prediction (hours, called intraday forecasting), used for safety operation in case of severe weather and also for the unbalanced situation; a medium-term prediction (days), used for production notification and maintenance; a long-term prediction (months), used for capacity planning, opportunity studies etc.

The prediction of wind generated power is dependent on the time scale. For instance, in the case of short-term prediction, stochastic methods (persistence, autoregressive models [12] and generalized equivalent Markov model [13]) are recommended. Furthermore, other research [14] has used the Kalman filter integrated with support vector regression (SVR) to obtain a $10 \%$ prediction improvement, comparing the obtained data with Artificial Neural Network (ANN) and autoregressive (AR).

Regarding the next day prediction, which is the purpose of this paper, a consistent approach is given by using ANN for wind energy production forecasting. In the case of ANN, various algorithms are explored $[15,16]$. In the presented case study, the Bayesian Regularization [17], Scalar Conjugate Gradient [18] and Levenberg-Marquardt [19] algorithms are explored. For ANN, the literature investigates the Back Propagatio neural network based on Particle Swarm Optimization [20, 21].

The studies [22,23, 25] reveal that a significant percentage of the energy forecasting error is given by the meteorological data input. The operational numerical weather prediction (NWP) models having relevance for wind power prediction in Europe are presented by the ANEMOS Plus project [5]. Furthermore, for meteorological forecasting, the wind speed prediction model and software (ENFOR, Forecast PRO, PredictWind) are presented in detail in $[22,23,24]$. The same studies reveal also that the error of these wind forecasting models, remains within $2.5 \%$ (in the case of large up-scaled German wind farms) or $15 \%$ MSE in the case of USA day-ahead forecasting and RMSE of $10 \%$ in the case of Ireland. Anyway, the forecasting accuracy of wind speed depends on time scale and resolution. In Romania, according to the official website of the National Administration for Meteorology, it uses global models (ECMWF and ARPEGE) as well as short range numerical weather prediction models (ALADIN, ALARO and COSMO) in its forecasts. According to the technical description of the Limited Area Models (LAM), the resolution varies from $2.8 \mathrm{~km}$ to 10 $\mathrm{km}$, the forecast horizon is up to 76 hours and the sampling frequency ranges from 1 to 3 hours. The presented ANN energy forecasting models must have a sampling frequency of 1 hour. The error 
types introduced by NWP are amplitude errors (the production time is correctly estimated, but the value of wind speed is underestimated or overestimated) and phase error (the value of the wind speed is accurate forecasted but the timing is not). The next chart shows the productionforecasting series for our case study, in the 10 MW wind production group, from $1^{\text {st }}$ to $3^{\text {rd }}$ of October 2015.

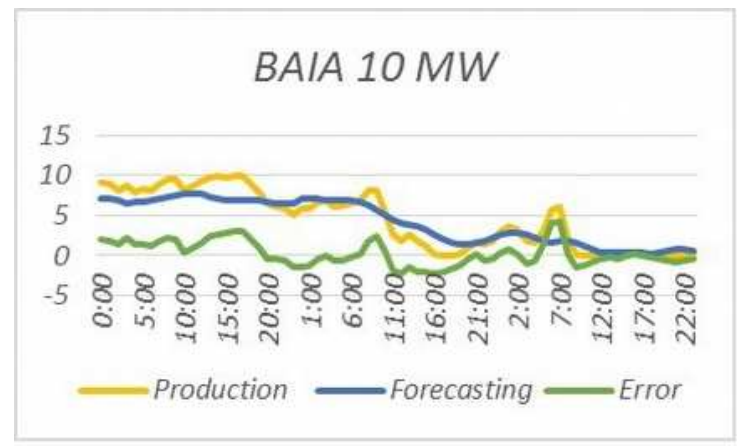

Figure 1. Production vs. forecasting Baia $10 \mathrm{MW}$ in the period $1^{\text {st }}-3^{\text {rd }}$ of October 2015

The computed errors of the on-site existing prediction system, provided by the Romanian company Wind Power Energy [24] for October 2015 is highlighted in the next figure.

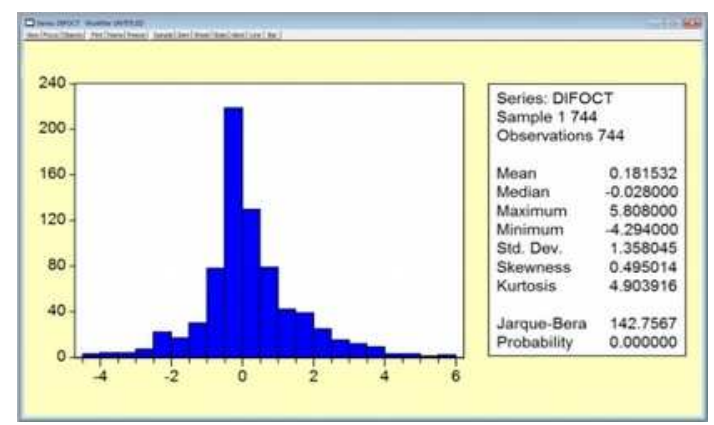

Figure 2. Error distribution for Baia 10MW in the period $1^{\text {st }}-31^{\text {st }}$ of October 2015

The upscaling algorithms are also explored by [5,23], general design principles and case studies, being depicted, mainly for off-shore or on-shore wind farms on flat terrain. According to [5], for hilly terrain, the energy forecast has the broader error dispersion when using existing software products. The paper emphasizes in the next sections, the Baia $5 \mathrm{MW}$ and the Baia $10 \mathrm{MW}$ case studies, that were conducted in collaboration with the Baia operator, in order to obtain a custom tailored forecasting solution of the produced energy. The forecasting solution took into account the influence of the hilly terrain (with $22.8 \mathrm{~m}$ level difference) and wind deflection (caused by the front hill, the distance between turbines being under the NWP resolution), over the turbines position. In the case of Baia $10 \mathrm{MW}$, we have forecasted using the upscaling algorithm applied to Baia $5 \mathrm{MW}$, aiming to obtain a better forecast accuracy than in [24].

\subsection{A solution for wind energy production forecasting using the ANN approach}

In our research project Intelligent system for predicting, analyzing and monitoring performance indicators and business processes in the field of renewable energies (SIPAMER) a computer based information system that targets the Romanian renewable energy market is to be developed. The system integrates data gathered from renewable power plants devices into a cloud computing database on top of which a forecast and analytical model that offers support for strategic business decisions is built (Figure 3):

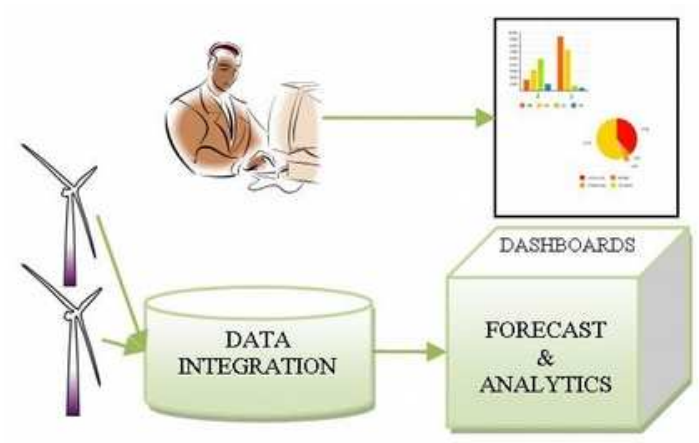

Figure 3. The SIPAMER system's components

The SIPAMER system is going to be developed on Java platform with business intelligence components for analyzing and reporting activities. The forecast model aims to minimize the prediction error and load the predictions into the analytical model to support the trading activities on the energy market. In order to build a suitable forecasting component for SIPAMER, we have developed, trained, validated and tested a series of artificial neural networks based on three algorithms: Levenberg-Marquardt (LM), Bayesian Regularization (BR) and Scalar Conjugate Gradient (SCG), using the Neural Network Toolbox from the development environment MatlabR2015a. After testing the solution, we have integrated the Matlab networks into SIPAMER by generating callable functions that incorporate the neural networks functionality that were further compiled as a Java package. Thus, the system can be accessed through a Java API, classes and interfaces being built for the analytics and reporting modules. Thus, wind power plants operators will use the 
web page interfaces having also export capabilities into various formats.

We have first identified the input parameters (the temperature, the absolute direction of the wind, the average wind speed) and the outputs (the wind energy production and the consumed energy) in order to develop our proposed forecasting solution. We have collected for the input and output parameters a number of 17,491 samples, obtained through direct measurements that were conducted hourly, over a two-year period (since $1^{\text {st }}$ January 2013 to $31^{\text {st }}$ December 2014), in a wind park located in Baia, in Tulcea County, in Romania.

The wind park where the measurements have been conducted comprises two types of turbines, Vestas V90 2MW/3MW IEC IA/IIA with a hub height of 90 meters, designed for high and medium wind sites with high turbulence. The cut-in wind speed for V90 2 MW is $4 \mathrm{~m} / \mathrm{s}$, while for V90 $3 \mathrm{MW}$ it is 3,5 $\mathrm{m} / \mathrm{s}$.. Taking into account the fact that the 5 MW production group comprises two turbines, we had to develop the neural networks taking into account 6 input parameters (the temperature, the absolute direction of the wind and the average wind speed for each turbine).

The 17,491 data samples that we have used when developing our forecasting solution have been accurately measured using the sensors of the turbines' equipment. However, in order to forecast both the wind energy production and the consumed energy, the wind park receives only a single set of NWP forecasted weather data from the National Administration for Meteorology that we are to use as data inputs for the 2MW-WEA01 turbine. Therefore, our neural networks solution must forecast at an initial stage the weather data corresponding to the 3MW-WEA02 turbine, located at a distance of $0.5189 \mathrm{~km}$ from the first turbine.

For this purpose, we have first developed, trained, validated and tested three neural networks for weather forecasting. These are the following: one network for each of the algorithms being used (LM, BR, SCG), with 3 weather data sets recorded by the sensors of the first turbine, as inputs (the temperature, the absolute direction of the wind, the average wind speed) and 3 weather data sets recorded by the sensors of the second turbine as outputs.

For each of the algorithms, we have used a sequential approach, in order to determine the optimal number of neurons in the hidden layer and after having performed several experimental tests (with the number of neurons ranging from 3 to 50), we have noticed that the best results were obtained by using 15 neurons in the hidden layer.

Thus, we have developed the weather forecasting neural networks based on the architecture that has offered the best prediction accuracy: 3 neurons for the Input data, 15 in the Hidden layer, 3 in the Output layer and 3 for the Output data (Figure 4).

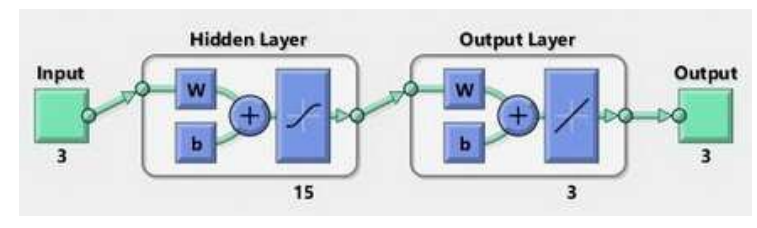

Figure 4. The architecture of the developed weather forecasting neural networks

In order to train, validate and test the neural networks that we have developed based on the three algorithms, we have divided the data set as follows: $70 \%$ was used for training the networks and $15 \%$ for testing purposes. The remaining $15 \%$ of data was used for the validation process in the cases of the LM and SCG algorithms, and it remained unallocated for the BR algorithm, as the validation step does not occur in the case of this algorithm.

We have decided to divide the data sets in this manner in order to obtain comparable sized data sets in all of the cases so that the final comparison of the obtained results becomes of outmost relevance. Also, this method of dividing the data sets has provided the best results for all of the developed networks. The contents of the data samples that make up each of the above-mentioned percentages were chosen randomly. We have tried to forecast the weather data by dividing beforehand the data set according to the months or seasons, thus obtaining monthly or seasonal weather forecasting neural networks, but the networks had a lower degree of prediction accuracy than a global weather forecasting neural network, obtained using the entire data set.

Comparing the performance plots corresponding to the networks (developed using the LM, BR and SCG algorithms), the error histograms and the regressions between the network targets and network outputs, we have concluded that the best results were achieved when using the weather forecasting 
neural network based on the BR algorithm (NetBaia5MWBRMeteoT2) (Table 1).

This network has provided the minimum value of the Mean Square Error (MSE), the best value of the correlation coefficient $\mathrm{R}$, the narrowest interval in which most of the errors fall and has also the advantage that avoids the overfitting process.

We have used the NetBaia5MWBRMeteoT2 weather forecasting neural network and we have predicted the temperature, the absolute direction of the wind, the average wind speed for the 3MW-WEA02 turbine. We have used the predicted data set in order to reconstruct the initial input data set that now contains the initial data for the first turbine and the predicted data for the second one.

Having obtained the reconstructed data set over a two-year period (since $1^{\text {st }}$ January 2013 to $31^{\text {st }}$ December 2014), we proceeded to the second stage of developing our forecasting solution. We have developed, trained, validated and tested 3 global energy forecasting neural networks (covering the 2-year period), based on the 3 algorithms, using the reconstructed data set as inputs and the wind energy production and the consumed energy as outputs. Through this method, we have obtained good prediction results. As we wanted to improve the obtained accuracy, we have divided the reconstructed data set into 4 smaller data sets, based on the four seasons specific to the temperate zone of Romania. We have used these seasonal data sets in order to develop 4 energy forecasting neural networks per each algorithm (LM, BR, SCG), resulting in a total number of 12 energy forecasting neural networks. By developing the seasonal solution, we have obtained improvements in the prediction results compared to the global approach, consisting in a simple energy forecasting neural network for the entire reconstructed data set.

Using an iterative testing procedure, we have determined for each of the algorithms, the appropriate number of neurons in the hidden layer. We have obtained the best results when using 12 neurons in the hidden layer. Thus, we have developed the energy forecasting neural networks based on the architecture that has offered the best results: 6 neurons for the Input data, 12 in the Hidden layer, 2 in the Output layer and 2 for the Output data (Figure 5).

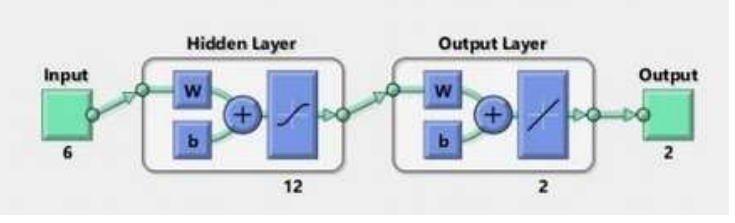

Figure 5. The architecture of the developed energy forecasting neural networks

We have divided the data set used for developing, validating and testing the energy forecasting neural networks in the same manner as in the case of the weather forecasting neural networks, developed in the first stage of the solution, as this approach proved to offer the best results.

The obtained results are summarized below, where MSE represents the value of the Mean Square Error and $\mathrm{R}$ the value of the correlation coefficient (Table 2).

Analysing Table 2, we have observed that the obtained results are comparable and highlight a high level of accuracy when forecasting the

Table 1. The performance indicators obtained when developing the weather forecasting neural networks

\begin{tabular}{|c|c|c|c|c|c|}
\hline No. & The developed network & The used algorithm & MSE & $\mathbf{R}$ & $\begin{array}{c}\text { The intervals in which most } \\
\text { of the errors fall }\end{array}$ \\
\hline $\mathbf{1}$ & NetBaia5MWLMMeteoT2 & LM & 0.090774 & 0.88623 & {$[-65.31 ; 67.97]$} \\
\hline $\mathbf{2}$ & NetBaia5MWBRMeteoT2 & BR & 0.082041 & 0.89369 & {$[-31.62 ; 35.16]$} \\
\hline $\mathbf{3}$ & NetBaia5MWSCGMeteoT2 & SCG & 0.083352 & 0.8921 & {$[-69.46 ; 93.48]$} \\
\hline
\end{tabular}

Table 2. The comparison between the results obtained using the LM, BR and SCG algorithms

\begin{tabular}{|c|c|c|c|c|c|c|}
\hline \multirow{2}{*}{ The prediction period } & \multicolumn{3}{|c|}{ MSE } & \multicolumn{3}{|c|}{ R } \\
\cline { 2 - 7 } & LM & BR & SCG & LM & BR & SCG \\
\hline 2 Years & 0.060908 & 0.057898 & 0.066406 & 0.9333 & 0.93474 & 0.92819 \\
\hline Spring & 0.048176 & 0.036081 & 0.050792 & 0.96637 & 0.96722 & 0.96195 \\
\hline Summer & 0.066927 & 0.059701 & 0.059853 & 0.95493 & 0.95735 & 0.94918 \\
\hline Autumn & 0.073134 & 0.068371 & 0.11224 & 0.94789 & 0.93749 & 0.95076 \\
\hline Winter & 0.059272 & 0.055915 & 0.063179 & 0.9603 & 0.96192 & 0.95458 \\
\hline
\end{tabular}


wind energy production and the consumed energy. Another important remark is that in most of the cases, the best results are provided by the seasonal approach and when using the networks developed based on the BR algorithm.

In the following, we present some of the experimental results and we analyse the obtained performance. As the seasonal approach has provided improved results compared to the global one, we present one of the seasonal sets of results, which takes into account the variations and particularities of the weather, during the different periods of the year.

The summer season has been randomly chosen together with the energy forecasting neural network based on the BR algorithm. In order to analyse the performance of this neural network, we have generated several plots that represent the best training performance (and the epoch at which it has been obtained), the errors histogram and also the regressions between the network targets and network outputs.

We have first analysed the training performance of the energy forecasting neural network based on the BR algorithm, developed for the Summer season, "NetBaiaVaraBR".

In the performance plot we have represented the train and test curves for the "NetBaiaVaraBR" network. We have obtained the best training performance at the $102^{\text {th }}$ epoch, when the value of MSE was 0.059701. In this case, the training and the test functions almost overlap and thus, the graphic highlights a high level of performance for this network.

A very important aspect that must be taken into account for is the overfitting phenomenon that produces large errors when new outputs are forecasted based on a new input data set. These errors occur even if the training of the network has been completed with very small errors. In this case, the network predicts very well the training set, but forecasts and generalizes very poor new input elements (Figure 6).

In order to overcome the overfitting issue and to improve the generalization process, we have implemented two solutions: the regularization and the early stopping.

The first technique, the regularization, is based on choosing a performance function as a linear combination between the sum of the squared errors and the sum of the squared weights. The second technique for improving the generalization is useful in the case of the LM and SCG algorithms and consists in partitioning the data set into three subsets for training, validation and testing purposes.

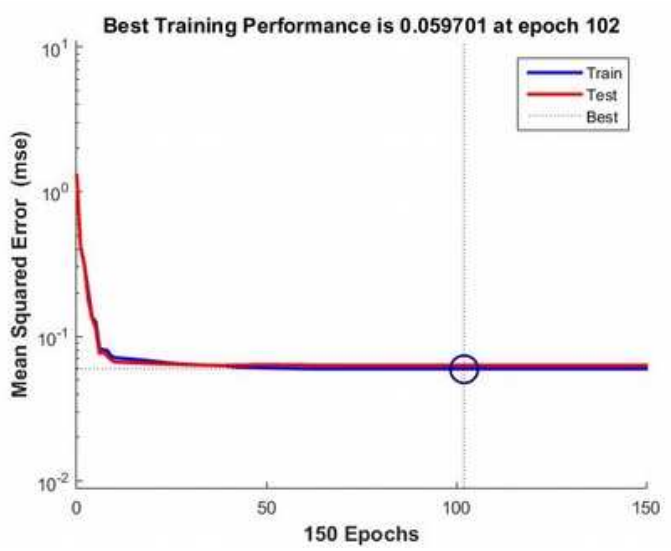

Figure 6. The training performance of the energy forecasting neural network based on the BR algorithm, developed for the Summer season

The training subset is useful for computing the gradient and for updating the network's biases and weights while the validation set helps in monitoring the error of the training process. When the training of the network begins, the validation error typically decreases along with the training set error. If the overfitting phenomenon does occur, the validation set error increases considerably. When the validation error has been increasing for a certain number of training epochs, the whole process is stopped, the local minimum of the validation error being represented by the last value recorded before these iterations. The weights and biases that correspond to the local minimum of the validation error are being returned.

The testing subset is useful for verifying that the data set has been divided correctly. The test set error is obtained and it is checked if the epoch in which it was recorded differs with a large margin from the one that corresponds to the minimum of the validation set error.We have configured the training process to stop if the mean square error has been increasing for six consecutive epochs in the case of the LM and SCG algorithms. For the BR algorithm, we have set the training process to continue until the convergence is reached. This means that the sum-squared weights, the sum-squared error and the number of parameters have become constant.

In the case of the "NetBaiaVaraBR" network, when we have verified the test and the validation curves, we have noticed that the test curve does 
not increase significantly before the training curve does and thus we can conclude that an overfitting process did not occur. This fact shows an appropriate partitioning of the data sets and an efficient training of the network.

Then we have represented and analysed the error histogram for the energy forecasting neural network based on the BR algorithm, developed for the summer season (Figure 7).

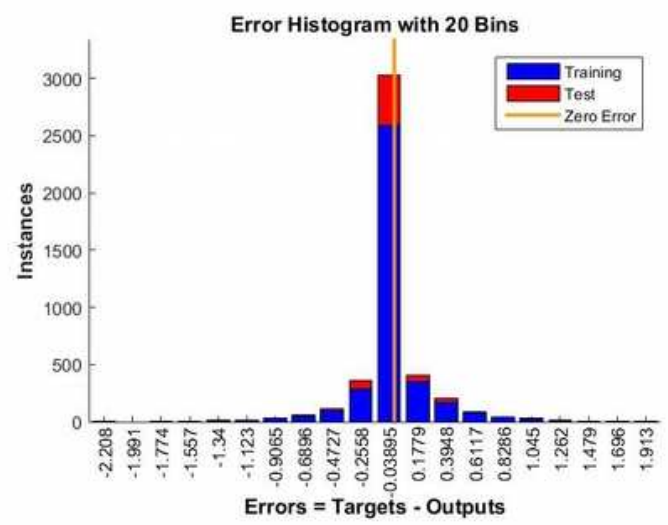

Figure 7. The error histogram for the energy forecasting neural network based on the BR algorithm, developed for the Summer season.

In the histogram, we have represented the testing data through the red bars and the training data through the blue bars. This representation is useful for obtaining information about the outliers (data points that do not fit as well as the majority of data). In the analysed case, most of the errors fall between -0.2558 and 0.1779 , but there are a few points whose errors fall outside this range.

The error histogram for the energy forecasting neural network based on the BR algorithm, developed for the summer season, confirms the fact that the obtained results are very good. We need to take into account the fact that our neural network forecasts two output parameters, with different orders of magnitude: the wind energy production varies between 0 and $5.007 \mathrm{MWh}$ and the consumed energy varies between 0 and $0.053 \mathrm{MWh}$. The above-depicted results confirm the efficiency and usefulness of a normalization process that we have applied. This process is useful when having to forecast multiple output parameters, having different orders of magnitude. If the network has not been submitted to a normalization process, it will forecast the first output element very well, while the other output elements will be poorly forecasted. In order to overcome this problem, we have applied a normalization process by programming the 'standard' value to the normalization performance parameter of the network. As a consequence, all the errors were computed as if the values of all the output parameters were ranging in the interval $[-1,1]$ and therefore, all the output elements were predicted with high accuracy. Afterwards, we have represented and analysed the regression plots between the network targets and network outputs, for the energy forecasting neural network based on the BR algorithm, developed for the summer season.

For the BR algorithm, we have represented three regression plots that highlight the regressions for the training, the testing and the whole data set. In these plots, the dashed lines represent an ideal, perfect fit, when the targets and the outputs coincide, while the solid line corresponds to the best linear regression. The regression plot shows that the correlation coefficient is greater than 0.95695 , in all the cases (the regressions for the training, the testing and the whole data set) (Figure 8).
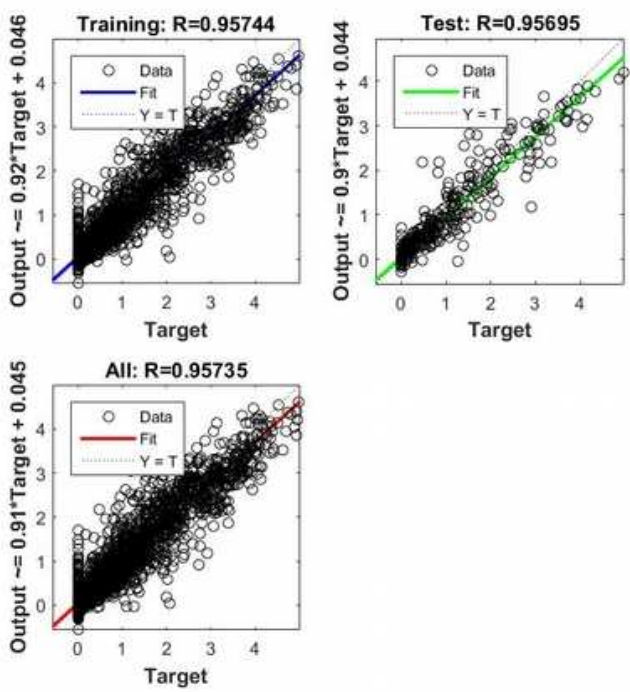

Figure 8. The regressions between the network targets and network outputs for the energy forecasting neural network based on the BR algorithm, developed for the summer season

Analysing the performance plots corresponding to the "NetBaiaVaraBR" neural network based on the BR algorithm, developed for the summer season, its error histogram and the corresponding regressions between the network targets and network outputs, we have concluded that the developed network has proved to offer a very good forecasting accuracy. This fact suits perfectly to our purpose, namely to obtain a 
forecasting solution for small size wind farms located on hilly terrain in Romania, based on artificial neural networks, using as input parameters the temperature, the absolute direction of the wind, the average wind speed and as outputs, the wind energy production and the consumed energy. Analyzing all the other global or seasonal networks, we have detected the same advantages, with minor differences regarding the provided performance.

\section{The Upscaling Process from 5 MW to 10 MW}

The Wind Power Plant (WPP) data is analyzed using SAS Enterprise Guide. We have analyzed 17491 records of hourly measured values for several months, such as: wind speed, wind direction, temperature for each wind generator.

The correlation coefficient between wind speed and output of WPP $10 \mathrm{MW}$ is 0.77 and the correlation coefficient between wind speed and output of WPP $5 \mathrm{MW}$ is 0.83 . The correlation coefficients between generated energy of WPP and temperature or wind direction are lower than 0.1 .

Comparatively, the two sites where the WPPs are located are in the same meteorological resolution area and the maximum distance between the turbines is less than the meteorological resolution. When we compared the output of both WPPs (Table 3), we noticed similarities between the operation of WPP 5 MW and WPP $10 \mathrm{MW}$.

Analyzing the recorded data during several months, we have observed that WPPs operate $20 \%$ of the time with $50 \%$ of installed power (Pi) and $20 \%$ of the time the WPPs do not operate. Only $2,8 \%$ of the time, WPPs operate at $95 \%$ of installed power. The correlation between the output of the WPP 10 MW and the output of the WPP $5 \mathrm{MW}$ is very strong (0.83632) mainly due to the location of WPPs (Figure 9).

Based on the strong correlation between the two WPPs, we proposed to forecast the output for the WPP 10 MW based on the estimated output of the WPP 5 MW. Therefore, we built a neural network with two hidden layers, the first layer with 30 neurons and the second with 20 neurons. The input data set contains records for the average wind speed for the two turbines of WPP
Table 3. Hourly average output of WPPs 5MW (10 MW)

\begin{tabular}{|l|r|}
\hline Mean & 1.455076831 \\
\hline Standard Error & $(2.54726788)$ \\
\hline Median & 0.011388065 \\
& $(0.02163513)$ \\
\hline Standard Deviation & 0.88 \\
& $(1.411)$ \\
\hline Sample Variance & 1.507316958 \\
& $(2.86361237)$ \\
\hline Kurtosis & 2.272004413 \\
& $(8.20027583)$ \\
\hline Skewness & -0.209085348 \\
& $(0.0929462)$ \\
\hline Range & 0.994940625 \\
& $(1.1043044)$ \\
\hline Minimum & 5.007 \\
& $(9.954)$ \\
\hline Maximum & 0 \\
\hline Sum & $(0)$ \\
\hline Count & 5.007 \\
\hline
\end{tabular}

$5 \mathrm{MW}$ and for the corresponding output. Our target is to estimate the output for WPP $10 \mathrm{MW}$.

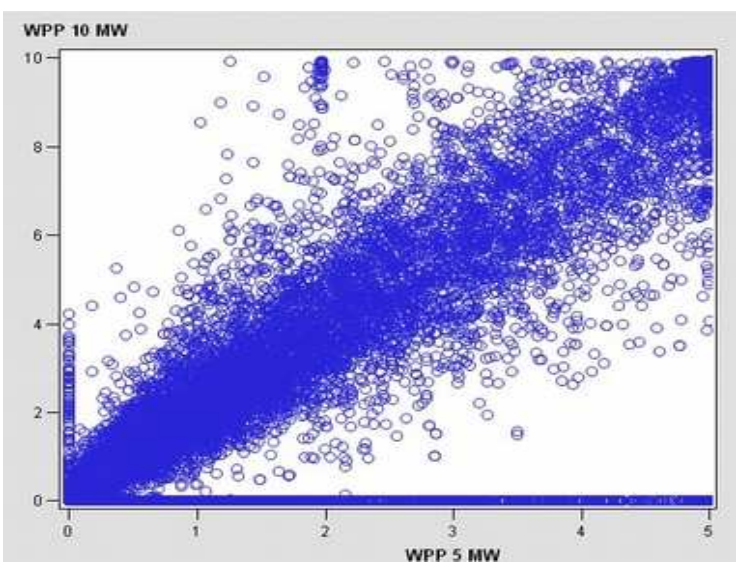

Figure 9. Scatter plot - correlation between WPP 5 MW and WPP $10 \mathrm{MW}$

After training the neural network, we have obtained a high level of performance, with a mean square error of 0.091754. Taking into account that the results have a good accuracy, we conclude that using an upscaling technique based on the data of one WPP, one can precisely forecast the output of the other WPP, because they are located in the proximity of each other.

Finally, using the same forecasted NWP, we have compared our solution to existing software [24], using the principle of comparison stated in [23], and we have obtained better results (decreasing standard error with $3,78 \%$ and lowering sample variance with $2.59 \%$ ) for BAIA $5 \mathrm{MW}$. In the case of 
BAIA 10MW, the prediction accuracy is slightly increased with an improvement of $0.78 \%$ regarding the MSE.

\section{Conclusions}

This paper presented the state of the art in wind energy production. The above-developed ANN model is part of the SIPAMER software product, designed for the wind power plant operator, in order to forecast the next day hourly energy production. Besides this feature, SIPAMER offers other various statistics and forecasts that could be used by existing operators and potential investors.

Our contribution to the state of the art consists in the improved forecasted results for small wind farms (BAIA 5MW), in comparison with the existing used software [24]. This improvement is the result of a two-step forecast technique, which best covers the situation of hilly terrain, with the wind direction changing from one turbine to another and the turbines being situated at different altitudes. Also, another contribution is the upscaling technique study case, which is slightly described in the literature.

We started from one turbine and we have forecasted the energy production for six turbines grouped into two production groups, located in hilly terrain conditions. The Romanian wind power energy market is characterised by the large number of small wind farms, most of them being located in hilly terrain.

The developed solution is also useful for the potential investors that intend to assess the potential of wind energy production in a certain geographical area that features hilly terrain.

Our proposed solution implies the costs of obtaining historical weather data for a certain area, but these costs are minor if compared to the extent of such an investment. After having being developed entirely, the SIPAMER information system will be offered for sale to the producers of renewable energy and will also be filed for a copyright registration to the Romanian Copyright Office.

The particularities for Romania derive from the dispatching and balancing system that is specific to the Romanian legislation. The reporting module of the SIPAMER information system is specific to the Romanian market and the technical prediction module can be used for any small production capacities situated on hilly terrain geographic areas.

\section{Acknowledgements}

This paper presents results of the research project: Intelligent system for predicting, analyzing and monitoring performance indicators and business processes in the field of renewable energies (SIPAMER), research project, PNII - Collaborative Projects, PCCA 2013, code 0996, no. 49/2014 funded by NASR.

\section{REFERENCES}

1. THE EUROPEAN WIND ENERGY ASSOCIATION Wind in power 2014 European Statistics, 2015 (http://www.ewea.org/fileadmin/files/librar y/publications/statistics/EWEA-AnnualStatistics-2014.pdf).

2. GLOBAL ENERGY COUNCIL, Wind in Number, 2015 (http://www.gwec.net/wpcontent/uploads/2015/03/GWEC_Global_ Wind_2014_Report_LR.pdf).

3. COMMISSION DECISION C (2011) 4938/2011 Green Certificates Promotion Scheme of Energy Produced from Renewable Energy Sources, European Commission.

4. LAW 220/2008, Law for Establishing the System for Promoting Energy from Renewable Sources, Romanian Parliament.

5. GIEBEL, G., R. BROWNSWORD, G. KARINIOTAKIS, M. DENHARD, C. DRAXL, The State-of-the-art in ShortTerm Prediction of Wind Power: A Literature Overview, ANEMOS plus, 2010 (http://www.anemosplus.eu/images/pubs/deliverables/aplus.deli verable_d1.2.stp_sota_v1.1.pdf).

6. BÂRA, A., I. LUNGU, S. V. OPREA, G. CĂRUȚAŞU, C. P. BOTEZATU, C. BOTEZATU, Design Workflow for Cloud Service Information System for Integration and Knowledge Management Based in Renewable Energy, Journal of Information Systems \& Operations Management, Volume 8, No.2/2014, pp. 230-237.

7. STĂNESCU, I. A., V. ŞTEFAN, G. NEAGU, C. E. CÎRNU, Renewable Energy Decision Support Systems: The 
Challenge of Data Integration, Studies in Informatics and Control, Vol. 24 (2), 2015, pp. 191-200.

8. http://www.transelectrica.ro/documents/101 79/32316/7productie19.pdf/3fd49432-

1dd5-46ea-bbd1-02964fe0b6c4, official website, accessed on 21.11.2015.

9. D'AMICO, G., F., PETRONI, F., PRATTICO, Wind Speed and Energy Forecasting at Different Time Scales: A Nonparametric Approach, Physica A: Statistical Mechanics and its Applications, Volume 406, 2014, pp. 59-66.

10. SHI, J., J. GUO, S., ZHENG, Evaluation of Hybrid Forecasting Approaches for Wind Speed and Power Generation Time Series, Renewable and Sustainable Energy Reviews, vol. 16(5), 2012, pp. 3471-3480.

11. LEI, M., L. SHIYAN, J. CHUANWEN, L., HONGLING, Z. YAN, A Review on the Forecasting of Wind Speed and Generated Power, Renewable and Sustainable Energy Reviews, Volume 13, Issue 4, 2009, pp. 915-920.

12. MAATALLAH, O. A., A. ACHUTHAN, K. JANOYAN, P. MARZOCCA, Recursive Wind Speed Forecasting Based on Hammerstein Auto-Regressive Model, App. Energy, vol. 145, 2015, pp. 191-197.

13. COSTA, A., A., CRESPO, J. NAVARRO, G. LIZCANO, H. MADSEN, E. FEITOSA, A Review on the Young History of The Wind Power Short-Term Prediction, Renewable and Sustainable Energy Reviews, vol. 12(6), 2008, pp. 1725-1744.

14. CHEN, K., J. YU, Short-Term Wind Speed Prediction Using an Unscented Kalman Filter Based State-Space Support Vector Regression Approach, App. Energy, vol. 113, 2014, pp. 690-705.

15. LIU, H., H. Q., TIAN, Y. F. LI, H. L. ZHANG, Comparison of Four Adaboost Algorithm Based Artificial Neural Networks in Wind Speed Predictions, Energy Conversion and Management, Volume 92, 2015, pp. 67-81.

16. CHITSAZ, H., N. AMJADY, H. ZAREIPOUR, Wind Power Forecast Using Wavelet Neural Network Trained by Improved Clonal Selection Algorithm,
Energy Conversion and Management, Volume 89, 2015, pp. 588-598.

17. BLONBOU, R., Very Short-Term Wind Power Forecasting with Neural Networks and Adaptive Bayesian Learning, Renewable Energy, Volume 36, Issue 3, 2011, pp. 1118-1124.

18. LI, G., SHI, J., On Comparing Three Artificial Neural Networks for Wind Speed Forecasting, Applied Energy, Volume 87, Issue 7, 2010, pp. 2313-2320.

19. OLAOFE, Z. O., A 5-Day Wind Speed \&Amp; Power Forecasts Using a Layer Recurrent Neural Network (LRNN), Sustainable Energy Technologies and Assessments, Vol. 6, 2014, pp. 1-24.

20. REN, C., N. AN, J. WANG, L. LI, B. HU, D. SHANG, Optimal Parameters Selection for BP Neural Network Based on Particle Swarm Optimization: A Case Study of Wind Speed Forecasting, Knowledge-Based Systems, Volume 56, 2014, pp. 226-239.

21. YEH, W. C., Y. M. YEH, P. C. CHANG, Y. C. KE, V. CHUNG, Forecasting Wind Power in the Mai Liao Wind Farm Based on the Multi-Layer Perceptron Artificial Neural Network Model with Improved Simplified Swarm Optimization, Intl. J. of El. Power \& En. Sys., vol. 55, 2014, pp 741-748.

22. FOLEY, A. M., P. G. LEAHY, A. MARVUGLIA, E. J. MCKEOGH, Current Methods and Advances in Forecasting of Wind Power Generation, Renewable Energy, Volume 37, no. 1, 2012, pp. 1-8.

23. KARINIOTAKIS, G., I. MARTÍ, D. CASAS, et al., What Performance Can Be Expected by Short-Term Wind Power Prediction Models Depending on Site Characteristics, In Proc. of the European Wind Energy Conference EWEC 2004.

24. Wind Power Energy, official website, www.wpe.ro, accessed on 13.11.2015.

25. MONTEIRO, C., R. BESSA, V. MIRANDA, A., BOTTERUD, A. J. WANG, G. CONZELMANN, Wind Power Forecasting: State-of-the-art 2009., No. ANL/DIS-10-1. Argonne National Laboratory. (ANL), 2009. 